\title{
Diagnosis of celiac disease and applicability of ESPGHAN guidelines in Mediterranean countries: a real life prospective study
}

\author{
Andrea Smarrazzo ${ }^{1,2}$, Zrinjka Misak ${ }^{3}$, Stefano Costa ${ }^{4}$, Dušanka Mičetić-Turk ${ }^{5}$, Mona Abu-Zekry ${ }^{6}$, Aydan Kansu, \\ Abdelhak Abkari ${ }^{8}$, Karim Bouziane-Nedjadi ${ }^{9}$, Mongi Ben Hariz ${ }^{10}$, Eleftheria Roma ${ }^{11}$, Virtut Velmishi ${ }^{12}$, \\ Maria Legarda Tamara $^{13}$, Thomas Attard ${ }^{14}$, Veselinka Djurisic ${ }^{15}$, Luigi Greco ${ }^{1,2}$ and Giuseppe Magazzü ${ }^{*}$
}

\begin{abstract}
Background: We assessed how the diagnosis of Celiac Disease (CD) is made and how the new ESPGHAN guidelines can be applied in children from countries with different resources.

Methods: A real life prospective study was performed in 14 centres of 13 different Mediterranean countries. Participants were asked to apply the usual diagnostic work-up for CD according to their diagnostic facilities.

Results: There were 1974 patients enrolled in the study, mean age 4 years, 10 months; 865 male, 1109 female. CD was confirmed in 511 (25.9\%) and was unconfirmed in 1391 (70.5\%) patients; 14 patients were diagnosed as having CD according to the new ESPGHAN guidelines, 43 patients were classified as having potential CD. In all participating countries the diagnosis of $C D$ relied on histology of duodenal biopsy; in 5 countries, HLA, and in one country endomysial antibodies (EMA) were not available. Symptoms did not add a significant increase to the pre-test probability of serological tests. The positive predictive value of tissue transglutaminase type 2 (tTG) antibodies performed with different kits but all corresponding to those recommended by ESPGHAN was $96.1 \%(95 \% \mathrm{Cl}$ 94-97.9\%) in presence of tTG>10xULN. In 135 patients with tTG >10xULN, HLA genotyping was performed and in all it was compatible with CD.
\end{abstract}

Conclusions: The results of our study show that CD diagnosis still relies on intestinal biopsy in the Mediterranean area. New ESPGHAN criteria are not applicable in 5 countries due to lack of resources needed to perform HLA genotyping and, in one country, EMA assay. Further simplification of the new ESPGHAN guidelines might be made according to what preliminarily the present results suggest if confirmed by new prospective studies.

Keywords: Celiac disease, Diagnosis, Mediterranean area, ESPGHAN

\section{Background}

Prevalence of celiac disease $(\mathrm{CD})$ has been estimated at around $1 \%$ in Western populations [1] but most patients remain undiagnosed. The burden of unrecognized $C D$ in countries with poor resources and facilities for diagnosis is very heavy [2]. Factors influencing the onset of this non communicable epidemics have been taken into consideration also recently [3-8]. New ESPGHAN guidelines [9]

\footnotetext{
* Correspondence: magazzug@unime.it

${ }^{4}$ Celiac Regional Centre, Pediatric Gastroenterology and Cystic Fibrosis Unit, University of Messina, Via Consolare Valeria 1, 98125, Messina, Italy Full list of author information is available at the end of the article
}

state that the protocol for the diagnosis of $C D$ changed as a result of the availability of $\mathrm{CD}$-specific tissue transglutaminase type 2 antibodies (tTG). As in children and adolescents with signs or symptoms suggestive of $\mathrm{CD}$ and high tTG titers with levels $>10$ times Upper Limits of Normal (ULN), the likelihood of villous atrophy (Marsh type 3 ) is very high, it has been suggested [9] that histological assessment may be omitted in symptomatic patients in whom these high tTG levels are verified by endomysial antibodies (EMA) positivity and are HLA-DQ2 and/or HLA-DQ8 heterodimer positive.

However ESPGHAN states that it is necessary to perform prospective research studies. 
After the publication of the new ESPGHAN criteria, apart from a large study investigating antibody diagnostics in paediatric CD [10], 2 prospective studies [11, 12] have been performed to our knowledge. One other prospective study adopted criteria other than those suggested by the guidelines [13].

Two main problems should be highlighted:

- there are many different tTG antibody tests;

- new ESPGHAN criteria were applied in some studies, but these studies have been performed, with only one exception [12], in tertiary centres of affluent countries in subjects with classical gastrointestinal disease.

Therefore, the conclusions of a recent commentary on the applicability of the new ESPGHAN Guidelines for diagnosing $C D$ in children from resource limited countries [14] rely on a single centre prospective study comprising only 142 children.

The Mediterranean Network for the Management of Food-Induced Diseases (MEDICEL) is a EUROMEDbased action in which Mediterranean countries with different resources and diagnostic facilities participate. It, therefore, represents the real life ideal setting in which the new ESPGHAN guidelines can be prospectively applied.

The objectives of this prospective study were to assess how the diagnosis of $\mathrm{CD}$ is made in different countries and how the new ESPGHAN guidelines can be applied in different Mediterranean countries.

\section{Methods}

\section{Study design}

A real life prospective study was performed in 14 centres of 13 different Mediterranean countries participating to the MEDICEL network; all unselected new cases referred to these Centres for suspected CD and asymptomatic subjects with autoimmune $\mathrm{CD}$-associated diseases or familiarity for CD were enrolled, from April 2013 to July 2014.

Participants were asked to apply the usual diagnostic work-up for $\mathrm{CD}$ according to their diagnostic facilities and to classify enrolled subjects as confirmed or unconfirmed $\mathrm{CD}$ according to shared criteria, as done in their usual clinical practice. Two sessions of shared agreement on diagnostic criteria were run through the MEDICEL network before starting the study.

Criteria for admission were: age below 18 years, clinical signs and symptoms of CD (systemic, gastrointestinal, extraintestinal) and/or associated autoimmune diseases (type 1 diabetes mellitus, thyroiditis, other autoimmune diseases) and/or no symptoms but familiarity for $\mathrm{CD}$ (1st and 2nd degree).

Criteria for exclusion were: already known diagnoses of CD only.

Familiarity, associated diseases, clinical symptoms, tTG as N x Upper Limit Normal (ULN), EMA, histology (Marsh-Oberhuber classification) [15], were collected into the database.

HLA-DQ2/DQ8 and follow-up were performed to confirm uncertain cases.

\section{Diagnostic procedures}

The tTG, EMA and HLA typing methods utilized by the Centres participating in the study, if available, are shown in Table 1.

tTG were determined with a kit of the 14 most frequently applied serum anti-TG2 IgA antibody assays taken in consideration in the new ESPGHAN guidelines (9).

Table 1 tTG, EMA and HLA typing methods utilized by the Centres participating in the study

\begin{tabular}{llll}
\hline Country & tTG kit & EMA substrate & HLA typing kit \\
\hline Albania & Orgentec & Monkey esophagus & NA \\
Algeria & Phadia - EliA Celikey & Monkey esophagus & NA \\
Croatia & Phadia - EliA Celikey & Monkey esophagus & NA LIfecodes Corporation \\
Egypt & Euroimmun & NA & Olerup, HLA typing kits \\
Greece & Inova & Monkey esophagus & BioDiagene - DQ-CD Typing Plus kit \\
Italy (ME) & Euroimmun & Umbilical cord & BioDiagene - DQ-CD Typing Plus kit \\
Italy (NA) & Eurospital & Monkey esophagus & Invitrogen / Life technologies \\
Malta & Orgentec & Monkey esophagus & Olerup, HLA typing kits \\
Montenegro & Aesku & Monkey esophagus & NA \\
Morocco & Orgentec & Monkey esophagus & Olerup, HLA typing kits \\
Slovenia & Eurospital & Monkey esophagus & Tepnel LIfecodes Corporation \\
Spain & Celikey; Pharmacia \& Upjohn & Monkey esophagus & NA \\
Tunisia & Inova & Monkey esophagus & Olerup, HLA typing kits \\
Turkey & Orgentec & Monkey esophagus & \\
\hline
\end{tabular}


With the exception of 1 centre, that utilized umbilical cord as substrate, all Centres assayed EMA on monkey esophagus as substrate.

In each Centre at least 4 endoscopic biopsy samples from duodenum, including one from bulbus, were taken.

Data provided by participants to the study were reevaluated by A.S., L.G. and G.M, and classified as shown in Table 2.

Crosstabs and stepwise statistics were generated by SPSS and t-Test, Relative Risk (RR) and Positive Predictive Value (PPV) were estimated for each variable assuming histology as the gold standard.

\section{Results}

\section{Population}

Demographic data of all the patients, and according to the final diagnosis, are shown in Table 3.

There were 1974 patients enrolled in the study, mean age 4 years, 10 months; 865 male, 1109 female. A global view of the classification and investigations performed by each Centre can be seen in the Flow Chart (Fig. 1) and in Table 4. CD was confirmed (CCD) in 511 (25.9\%) and was unconfirmed (UCD) in 1391 (70.5\%) patients. Apart from 14 patients diagnosed as having $\mathrm{CD}$ according to the new ESPGHAN guidelines (NCCD: New Criteria Celiac Disease), $2.47 \%$ of the final amount of diagnoses of CD, 43 patients were classified as having Potential Celiac Disease (PCD), 7.4\% of the CD population, and 16 defined as having "high probability" of CD, as EMA or HLA were positive, but not both were done.

\section{Symptoms}

The proportion of asymptomatic cases enrolled for familiarity and of symptoms in CCD and UCD are shown in Table 5. A higher prevalence of asymptomatic cases, food refusal, globose abdomen and paleness was found in CCD, whereas abdominal pain and constipation were more common in the UCD. No difference was found for diarrhoea and failure to thrive between the two groups.

\section{Diagnostic tools}

Table 4 shows the frequency of various investigations performed in different countries: in 5 countries, HLA, and in one EMA assays were not available. HLA was not performed in Malta because it was not considered necessary.

\section{Serology}

In 91 patients, tTG antibodies were not performed; out of these, 7 patients showed positivity for EMA and histology, allowing to classify them as CCD, while in the other 84 cases $\mathrm{CD}$ was excluded (thus, they were classified as UCD, see Fig. 1). Of 1883 patients evaluated for tTG: 1219 were negative, while 664 were positive.

Among the 1307 UCD patients in whom tTG were performed, 1215 resulted negative, while 92 resulted positive: 70/1307 (5.4\%) had tTG titre higher than $2 \times$ ULN, and 22/1307 (1.7\%) had tTG titre higher than $10 \times$ ULN.

Four patients received diagnosis of $\mathrm{CD}$ despite having negative titre of tTG in presence of EMA positve: one was classified as PCD because of Marsh type 0, while the other 3 presented a Marsh type equal to or higher than 2. tTG sensitivity and specificity were $99.3 \%$ (95\% CI 98.6-99.9\%) and 93\% (95\% CI 91.6-94.3\%) respectively, with a RR 1888.53 (95\% CI 690.61-5164.35) of being celiac.

Results of EMA assay are shown in Fig. 2. EMA sensitivity and specificity were $99.5 \%$ (95\% CI 98.8-100\%) and 90.1\% (95\% CI 87.5-92.7\%) respectively.

Both EMA and tTG were performed in 464 patients who underwent intestinal biopsy as well: 66 were EMA negative and 398 EMA positive. The reliability of tTG versus EMA is defined by its sensitivity $95.7 \%$ (95\% CI 93.86-97.54\%) and specificity 95.8\% (95\% CI 93.91-97.69\%). High titers of tTG $>10 \times$ ULN were found in 427 patients, out of whom EMA were performed in 289 and were found absent in only 3 patients (1.04\%). Intestinal biopsy was performed in 2 of these latter patients and in both, histology showed a Marsh type 3c confirming CD diagnosis. The third was classified as UCD because of having done neither HLA nor intestinal biopsy.

Table 2 Classification of the patients' diagnoses

\begin{tabular}{lllll}
\hline Classification & Histology & tTG & EMA & HLA \\
\hline CCD $^{a}$ & $\geq$ Marsh type 2 & Positive or not done & Positive or not done & Positive or not done \\
NCCD & Not done & $>10 \times$ ULN & Positive & Positive \\
UCD & Not done or Marsh type 0-1 & $<5 \times$ ULN & Negative & Not done \\
PCD & Marsh 0-1 & Positive & Positive & Positive \\
High probability CD & Not done & $>10 \times$ ULN & Positive or not done & Positive or not done
\end{tabular}

$C D$ Celiac Disease, CCD Confirmed Celiac Disease, NCCD New Criteria Celiac Disease, UCD Unconfirmed Celiac Disease, $P C D$ Potential Celiac Disease, $t T g$ tissue transglutaminase type 2 antibodies, EMA endomysial antibodies, ULN Upper Limit of Normal

${ }^{a}$ at least tTG or EMA positive or both positive; ${ }^{b}$ EMA or HLA positive but not both done. This category was not taken into account for comparison of the variables considered in the study 
Table 3 Demographic data according to the final diagnosis

\begin{tabular}{lllr}
\hline & No. $(\%)$ & Gender (F/M) & Age (M \pm SD) \\
\hline PATIENTS & 1974 & $1109 / 865$ & $4.83 \pm 4.72$ \\
Confirmed & $511(25.9 \%)$ & $320 / 191$ & $4.1 \pm 4.11$ \\
Unconfirmed & $1391(70.5 \%)$ & $739 / 652$ & $5.11 \pm 4.89$ \\
Potential & $42(2.1 \%)$ & $28 / 14$ & $4.25 \pm 4.51$ \\
Diagnosed according new ESPGHAN guidelines & $14(0.7 \%)$ & $10 / 4$ & $3.97 \pm 3.59$ \\
High probability & $16(0.8 \%)$ & $12 / 4$ & $2.98 \pm 2.93$ \\
\hline
\end{tabular}

In 135 patients with $\mathrm{tTG}>10 \mathrm{x}$ ULN, HLA genotyping was performed and in all of them it was compatible with $\mathrm{CD}$.

Out of the 413 patients with tTG > $10 \times$ ULN (excluding 14 patients diagnosed according to the new ESPGHAN criteria) both EMA and HLA were performed in 88. In all these patients a duodenal biopsy was performed, and in 85/88 the diagnosis would have been correctly made according to the new criteria; in 3 (3.41\%) the diagnosis of PCD would have been missed in absence of the biopsy.

Biopsy was performed in 382 out of the 427 patients with high titre tTG, and histology showed at least a Marsh type 2 in 367 patients (96.1\%). Thus, in this subgroup of patients, the positive predictive value of tTG > 10 x ULN was 96.1\% (95\% CI 94-97.9\%).

\section{Biopsy}

Of 1974 cases included in this study, 1002 underwent an intestinal biopsy and in 542 at least Marsh type 2 mucosal damage was found: 511 (94\%) were classified as CCD, because of positivity of tTG or EMA, 31 (6\%) were classified as UCD because of negativity of both tTG and EMA.

Out of 460 patients with Marsh type 0-1, 42 (9\%) were classified as PCD in light of the positivity of EMA or tTG antibodies. In particular, 8 of them had tTG $>10 \mathrm{x}$ ULN, 6 between 5 and $10 x$ ULN, 27 between 1 and $5 x$ ULN. One patient had a negative titre of tTG (0.9 x ULN), but he was symptomatic (abdominal pain) and had positive EMA with compatible HLA.

In 364/460 with a Marsh type 0-1 at biopsy, no antibodies were produced and they were classified as UCD.

Overall, 568 patients were diagnosed as having CD (including CCD, NCCD and PCD), $2.47 \%$ of whom according to the new guidelines.

The presence of high titre of tTG correlates with villous atrophy (Fig. 3).

\section{HLA}

Less than $20 \%$ (382) of the total number of enrolled patients underwent a HLA analysis: in 63, that were both DQ2 and DQ8 negative, CD was finally excluded they were classified as UCD.

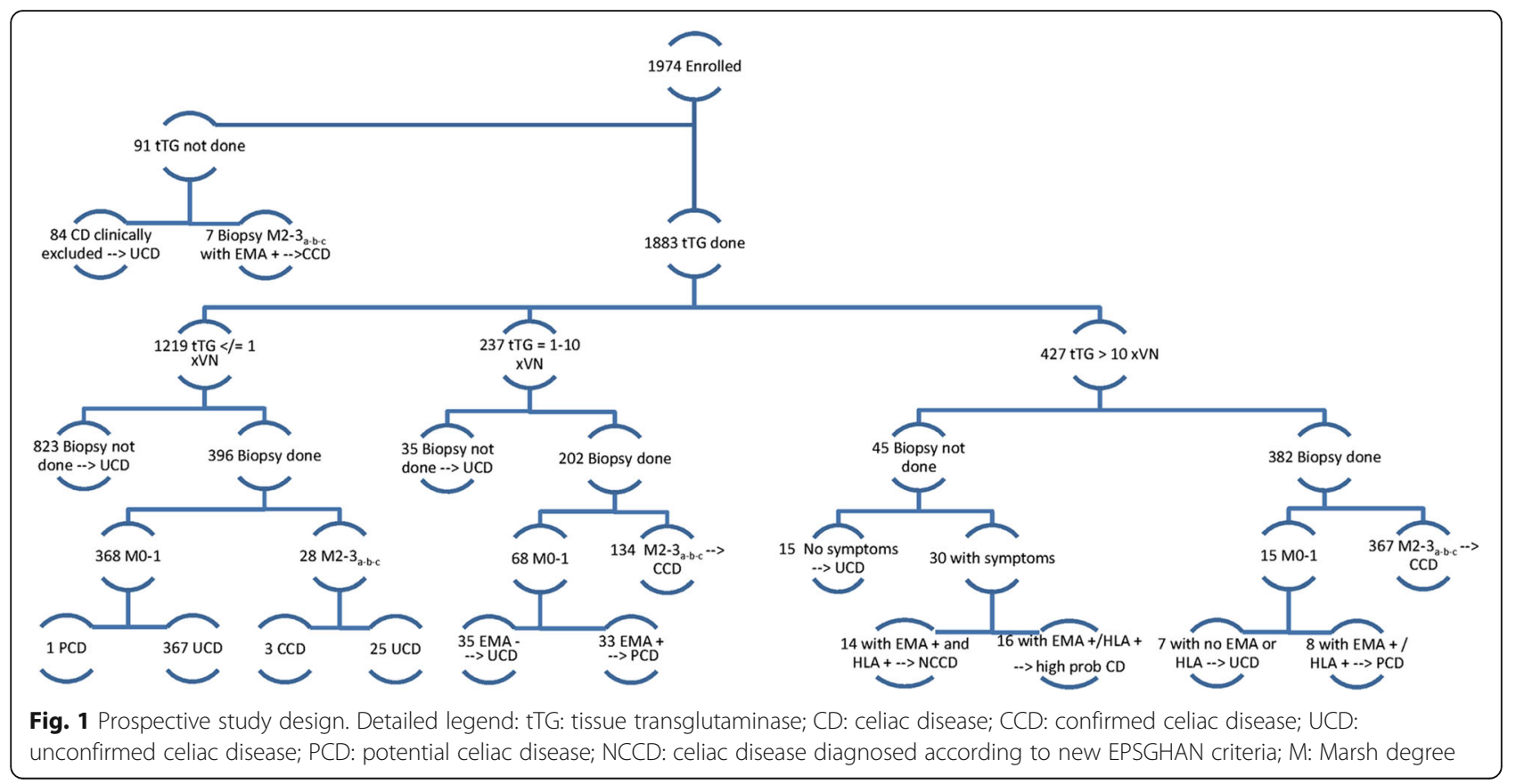


Table 4 Distribution of patients by study centers and according to the final diagnosis and performed diagnostic investigations

\begin{tabular}{|c|c|c|c|c|c|c|c|c|}
\hline Country & N. Pts. & $\begin{array}{l}\text { N. CCD + } \\
\text { NCCD }^{\mathrm{a}}\end{array}$ & N. UCD & N.PCD & N. tTG & $\begin{array}{l}\text { N. EMA } \\
\text { (in CCD) }\end{array}$ & $\begin{array}{l}\text { N. Intestinal } \\
\text { biopsy (in CCD) }\end{array}$ & N. HLA \\
\hline Albania & 45 & 25 & 20 & 0 & 45 & $7(1)$ & $32(25)$ & 0 \\
\hline Algeria & 71 & 17 & 54 & 0 & 70 & $6(2)$ & $54(17)$ & 0 \\
\hline Croatia & 310 & $12+1$ & 293 & 3 & 304 & $25(6)$ & $202(12)$ & 14 \\
\hline Egypt & 200 & 5 & 195 & 0 & 159 & 0 & $21(5)$ & 0 \\
\hline Greece & 46 & 35 & 10 & 1 & 46 & $40(31)$ & $46(35)$ & 27 \\
\hline Italy (ME) & 332 & 111 & 199 & 15 & 307 & $273(108)$ & $142(111)$ & 13 \\
\hline Italy (NA) & 339 & $152+9$ & 153 & 17 & 330 & 281 (151) & $202(152)$ & 109 \\
\hline Malta & 27 & 14 & 13 & 0 & 26 & $16(14)$ & $27(14)$ & 0 \\
\hline Montenegro & 18 & 11 & 4 & 2 & 17 & $5(3)$ & $16(11)$ & 11 \\
\hline Morocco & 106 & 45 & 61 & 0 & 100 & $1(1)$ & $90(45)$ & 0 \\
\hline Slovenia & 234 & $29+1$ & 203 & 1 & 234 & $234(29)$ & $34(29)$ & 66 \\
\hline Spain & 40 & $4+3$ & 32 & 1 & 40 & $10(4)$ & $8(4)$ & 9 \\
\hline Tunisia & 67 & 22 & 44 & 1 & 66 & $23(11)$ & $43(22)$ & 0 \\
\hline Turkey & 139 & 29 & 109 & 1 & 139 & $5(1)$ & $85(29)$ & 133 \\
\hline TOTAL & 1974 & $511+14$ & 1391 & 42 & 1883 & 926 (362) & $1002(511)$ & 382 \\
\hline
\end{tabular}

N. Pts Number of Patients, N. CCD Number of Patients with Confirmed Celiac Disease, N. UCD Number of Patients with Unconfirmed Celiac Disease, N. PCD N. of Patients with potential celiac disease, N. tTG Number of tissue TransGlutaminase antibody assays, N. EMA Number of EndoMysial Antibody assays

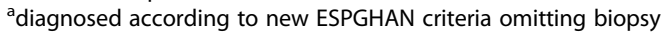

\section{Associated diseases}

About $80 \%$ of the total study population had no other disease. The significant differences in prevalence of associated diseases in UCD and CCD are shown in Table 6. The frequency of thyroiditis, type 1 diabetes mellitus and dermatitis herpetiformis was higher in CCD, whereas IgA deficiency and hypertransaminasemia were higher in UCD.

\section{Discussion}

Our study, for the first time, provides a wide picture of CD diagnosis feasibility in 13 Mediterranean countries with different health resources and facilities. It is also the largest one investigating antibody diagnostics in

Table 5 Distribution of symptoms in UCD and CCD patients

\begin{tabular}{lllll}
\hline Symptoms & UCD (\%) & CCD (\%) & $x^{2}$ & $p$ \\
\hline No symptoms & $93(6.7 \%)$ & $120(20.6 \%)$ & 82.4 & $<0.0001$ \\
Abdominal pain & $402(28.9 \%)$ & $69(11.8 \%)$ & 65.9 & $<0.0001$ \\
Constipation & $89(6.4 \%)$ & $17(2.9 \%)$ & 9.8 & 0.002 \\
Diarrhea & $297(21.4 \%)$ & $145(24.9 \%)$ & 2.93 & 0.087 \\
Failure to thrive & $266(19.1 \%)$ & $98(16.8 \%)$ & 1.46 & 0.227 \\
Food refusal & $16(1.2 \%)$ & $14(2.4 \%)$ & 4.3 & 0.038 \\
Globose abdomen & $29(2.1 \%)$ & $22(3.8 \%)$ & 4.66 & 0.031 \\
Mood changes & $5(0.4 \%)$ & $6(1 \%)$ & 3.33 & 0.068 \\
Paleness & $46(3.3 \%)$ & $31(5.3 \%)$ & 4.43 & 0.035 \\
Vomiting & $103(7.4 \%)$ & $30(5.1 \%)$ & 3.34 & 0.068 \\
Others & $45(3.2 \%)$ & $31(5.3 \%)$ & 4.81 & 0.028 \\
\hline
\end{tabular}

UCD Unconfirmed Celiac Disease, CCD Confirmed Celiac Disease paediatric CD that prospectively evaluates the new ESPGHAN guidelines in different countries including those with poor resources.

In all participating countries, the diagnosis of $\mathrm{CD}$ relied on histology of duodenal biopsy, but other diagnostic procedures were not always available. In particular, in 5 countries, HLA, and in 1, EMA were not performed. Even though HLA was not required when the diagnosis was not in doubt, in some countries the limitation of performing it derives from its cost. The same is true for EMA assay.

On the other hand, in order to apply what is suggested by the new ESPGHAN guidelines, apart from the presence of symptoms and high antibody levels, HLA has to be compatible and EMA have to be present if the diagnosis of $C D$ is to be made without a biopsy. In 4 centres, the diagnosis of $\mathrm{CD}$ was made for a total of 14 patients according to these new guidelines, omitting a duodenal biopsy.

Even though $\mathrm{CD}$ can be reliably diagnosed following the latest ESPGHAN and BSPGHAN guidelines [16], as not all diagnostic procedures required by the new guidelines are available in all countries, our results may suggest to further simplify the new ESPGHAN guidelines. As a matter of fact, it is not useful nor necessary to perform an expensive determination, such as HLA, as in all patients with tTG higher than $10 \mathrm{x}$ ULN who had HLA heterodimer determined it was compatible with $\mathrm{CD}$. Moreover EMA, which is not easily performed in all countries, does not add diagnostic accuracy to tTG, as 


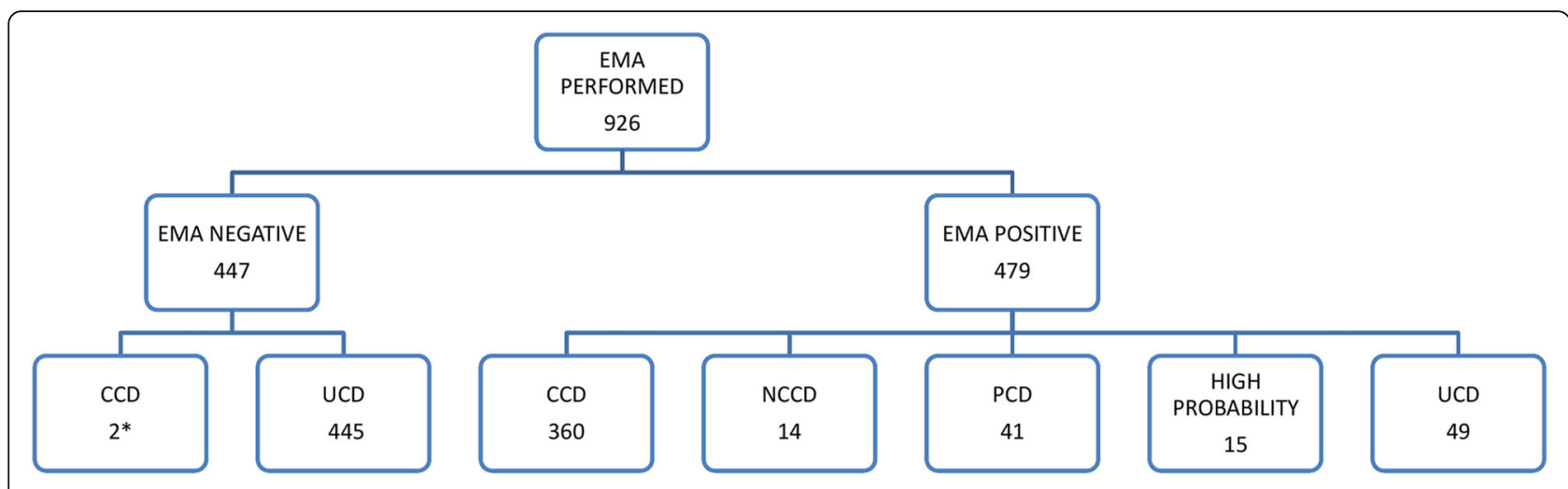

Fig. 2 Role of EMA assay in the diagnosis. Detailed legend: * ${ }^{t} \mathrm{Tg}$ and Histology positive

suggested by the cases in which both tests were performed and tTG was $>10 \times$ ULN. In 3 cases of our study, in whom EMA were negative, histology showed a picture that allowed us to make the diagnosis of $\mathrm{CD}$. Rather than determining EMA, in the presence of such a high titre tTG, it is better to repeat a second test for tTG according also to what is suggested by the Joint BSPGHAN and Coeliac UK guidelines for diagnosis and management of $\mathrm{CD}$ in children [17]. In a commentary regarding applicability of the new ESPGHAN guidelines for diagnosing celiac disease in children from resource limited countries [14], it has been suggested that positive HLA-DQ2/DQ8 serotype and EMA are necessary in order to apply the ESPGHAN guidelines for serological diagnosis of $C D$, and that $C D$ should not be diagnosed on the basis of a single high tTG-titre. Our study comprising many countries with limited resources suggests that both HLA and EMA may be omitted and CD may be diagnosed on the basis of repeated high tTG titres. Altogether, the diagnosis would have been correctly made according to the new criteria in 85 out 88 patients with tTG $>10 \times$ ULN who underwent tTG and EMA determination, together with intestinal biopsy. In 3 patients (3.41\%), the diagnosis of $\mathrm{PCD}$ would have been missed in absence of the biopsy.

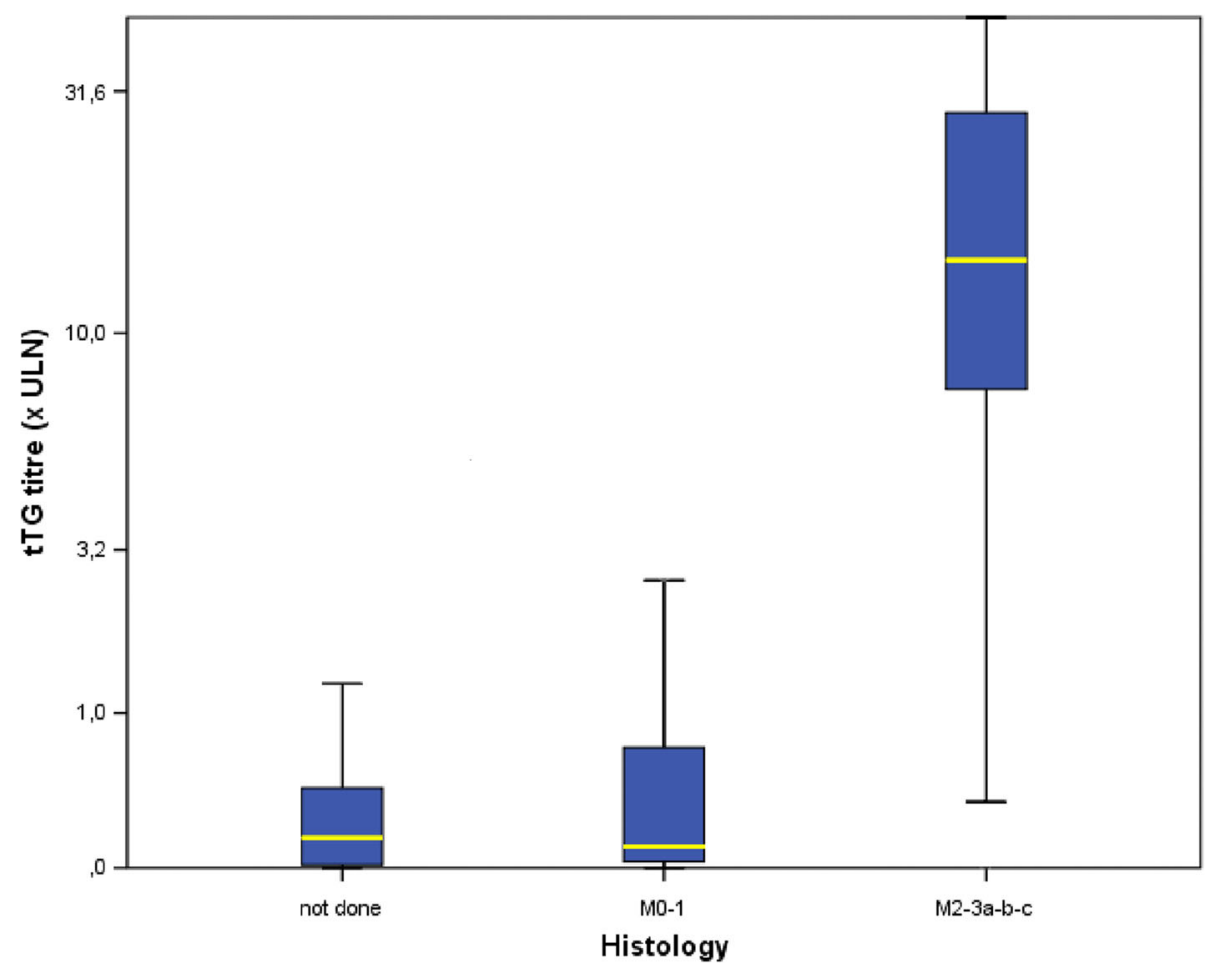

Fig. 3 Correlation among tTG titre and villous atrophy 
Table 6 Significant differences in prevalence of associated diseases in unconfirmed celiac disease (UCD) and confirmed celiac disease (CCD)

\begin{tabular}{lllll}
\hline Associated diseases & UCD (\%) & CCD (\%) & $x^{2}$ & $p$ \\
\hline Thyroiditis & 1 & 5.7 & 38.7 & 0.0001 \\
Type 1 Diabetes Mellitus & 7 & 3 & 16 & 0.0001 \\
Dermatitis herpetiformis & 0.1 & 1.5 & 17.1 & 0.0001 \\
IgA deficiency & 1.8 & 0.3 & 6.3 & 0.012 \\
Hypertransaminasemia & 2.7 & 0.7 & 7.87 & 0.005 \\
\hline
\end{tabular}

Thus, some practical issues need to be addressed. In adopting the new ESPGHAN criteria, it has to be kept in mind that there is a chance that patients might have a potential CD. In a prospective cohort study [18], which describes the long term natural history of potential $C D$ by a 9 years follow-up, the risk of becoming atrophic was estimated at $18 \%$ especially in subjects with persistent positive serology, while serology became negative in $20 \%$ of potential patients on follow up. Even if rarely, potential celiac patients showed a TTG value $>10 \times$ ULN: hence this limit does not exclude the chance of finding potential celiac cases. Starting from this point, the decision to omit biopsy and the chance of being a potential CD should be thoroughly discussed with families considering the life-long diagnosis of CD. The new ESPGHAN guidelines also state that it is important to be precise in the clinical evaluation of patients and to perform prospective research studies. To the best of our knowledge, our prospective study is the largest one including 511 $(+14)$ CD patients and 1391 controls and it shows that symptoms are not able to assign a pre-test probability to serological tests. On the other hand, Webb et al. [11] found no difference in terms of diagnostic accuracy of tTG in asymptomatic children diagnosed as having CD detected by screening.

We are aware of the limitations of our study, mostly due to the unequal distribution of enrolled patients across participating countries, although the total number is the largest one reported.

Therefore, further prospective multicentre studies with an homogeneous enrolment of patients should be planned also in order to try a further simplification of ESPGHAN guidelines.

Moreover, due to different test kinetics the $10 \mathrm{x}$ UNL is not the same with all tTG tests. However, all the centres participating in the study utilized one of the 14 most frequently applied serum tTG assays in Europe taken in consideration in the new ESPGHAN guidelines Appendix. All these assays underwent United Kingdom National External Quality Assessment according to which the $10 \mathrm{x}$ ULN was suggested by the new guidelines in order to omit intestinal biopsy. Considering that all the centres took at least 4 duodenal biopsy samples always including a sample from the bulbus we are confident that histology is the right gold standard chosen in this study.

\section{Conclusions}

This is the largest prospective study providing a wide picture of $\mathrm{CD}$ diagnosis feasibility in Mediterranean countries with different health resources and facilities. The results of our study show that CD diagnosis still relies on intestinal biopsy in the Mediterranean area. New ESPGHAN criteria are not applicable in 5 countries due to lack of resources needed to perform HLA genotyping and, in one country, EMA assay. Further simplification of the new ESPGHAN guidelines might be made according to what preliminarily the present results suggest if confirmed by new prospective studies. This would be a result of great value especially for countries with limited resources, even though the chance of a potential CD has to be taken into account and discussed with the families when intestinal biopsy is omitted.

\section{Abbreviations}

BSPGHAN: British society for pediatric gastroenterology, hepatology and nutrition; CCD: Confirmed celiac disease; CD: Celiac disease; ESPGHAN: European society for pediatric gastroenterology, hepatology and nutrition; NCCD: New criteria celiac disease; PCD: Potential celiac disease; PPV: Positive predictive value; RR: Relative risk; tTG: tissue transglutaminase type 2 antibodies; UCD: Unconfirmed celiac disease; ULN: Upper limit of normal

\section{Acknowledgement}

We thank Trays MacDonnell for revising the English of the article.

\section{Funding}

Italian Department of Health, Direction of International Affairs; Euromed action. Project: MEDICEL-Mediterranean Network for Celiac Disease-Phase II" (CUP n E61J1 1000450001); ELFID - European Laboratory for Food Induced Disease.

\section{Availability of data and materials}

Blinded data of patients were collected into a web-based database of the MEDICEL Mediterranean Network (www.medicel.unina.it/). Raw data are available upon request to the corresponding author.

\section{Authors' contributions}

AS, LG and GM conceptualized and designed the study, carried out the initial analyses, drafted the initial manuscript, and approved the final manuscript as submitted. ZM drafted the initial manuscript, reviewed and revised the manuscript and approved the final manuscript as submitted. SC, DM-T, MA-Z, AK, AA, KB-N, MBH, ER, W, MLT, TA and VD reviewed and revised the manuscript, and approved the final manuscript as submitted. All authors approved the final manuscript as submitted.

\section{Competing interests}

The authors declare that they have no competing interests.

\section{Consent for publication}

Not applicable.

\section{Ethics approval and consent to participate}

The Ethics Committee of the University Hospital of Messina, where the Coordinating Centre is allocated, approved the study (Prot. E 35/12) and extended the approval to all the centers participating in the study according to the 2008, March 28th statement of the Italian Drug Agency (AIFA) on observational studies. Parent written consent was obtained in all the Centers participating in the study as suggested by ethical institutional review boards. 


\section{Authors' information}

Not applicable.

\section{Author details}

'Department of Translational Medical Sciences, School of Medicine, University "Federico II", Naples, Italy. European Laboratory for Food Induced Diseases, Naples, Italy. ${ }^{3}$ Children's Hospital Zagreb, Zagreb, Croatia. ${ }^{4}$ Celiac Regional Centre, Pediatric Gastroenterology and Cystic Fibrosis Unit, University of Messina, Via Consolare Valeria 1, 98125, Messina, Italy. ${ }^{5}$ Paediatric Department, University Medical Centre, Maribor, Slovenia. ${ }^{6}$ Cairo University Children Hospital, El Cairo, Egypt. ${ }^{7}$ Department of Pediatric Gastroenterology, School of Medicine, Ankara University, Ankara, Turkey. ${ }^{8}$ Centre Hospitalier Universitaire Ibnou Rochd, Casablanca, Morocco. ${ }^{9}$ Faculté de Médecine d'Oran, Oran, Algeria. ${ }^{10}$ Pediatric Unit, Mongi SLIM's Hospital of Tunis, Marsa, Tunisia. ${ }^{11}$ First Department of Pediatrics, University of Athens, Athens, Greece. ${ }^{12}$ Service of Pediatric Gastroenterology "Mother Teresa" Hospital, Tirana, Albania. ${ }^{13}$ Paediatric Gastroenterology Unit, Cruces University Hospital, Bilbao, Spain. ${ }^{14}$ University of Malta, Msida, Malta. ${ }^{15}$ Clinical Center of Montenegro, Podgorica, Montenegro.

Received: 6 June 2016 Accepted: 18 January 2017

Published online: 21 January 2017

\section{References}

1. Green PH, Cellier C. Celiac disease. N Engl J Med. 2007;357:1731-43.

2. Greco L, Timpone L, Abkari A, Abu-Zekry M, Attard T, Bouguerrà F, Cullufi $P$, Kansu A, Micetic-Turk D, Mišak Z, Roma E, Shamir R, Terzic S. Burden of celiac disease in the Mediterranean area. World J Gastroenterol. 2011;17: 4971-8.

3. Emilsson L, Magnus MC, Størdal K. Perinatal risk factors for development of celiac disease in children, based on the prospective Norwegian Mother and Child Cohort Study. Clin Gastroenterol Hepatol. 2015;13:921-7.

4. Mårild K, Stephansson O, Montgomery S, Murray JA, Ludvigsson JF. Pregnancy outcome and risk of celiac disease in offspring: a nationwide case-control study. Gastroenterology. 2012;142:39-45.

5. Lionetti E, Castellaneta S, Francavilla R, Pulvirenti A, Tonutti E, Amarri S, Barbato M, Barbera C, Barera G, Bellantoni A, Castellano E, Guariso G, Limongelli MG, Pellegrino S, Polloni C, Ughi C, Zuin G, Fasano A, Catassi C, SIGENP (Italian Society of Pediatric Gastroenterology, Hepatology, and Nutrition) Working Group on Weaning and CD Risk. Introduction of gluten, HLA status, and the risk of celiac disease in children. N Engl J Med. 2014; 371:1295-303.

6. Vriezinga SL, Auricchio R, Bravi E, Castillejo G, Chmielewska A, Crespo Escobar P, Kolaček S, Koletzko S, Korponay-Szabo IR, Mummert E, Polanco I, Putter H, Ribes-Koninckx C, Shamir R, Szajewska H, Werkstetter K, Greco L, Gyimesi J, Hartman C, Hogen Esch C, Hopman E, Ivarsson A, Koltai T, Koning F, Martinez-Ojinaga E, te Marvelde C, Pavic A, Romanos J, Stoopman E, Villanacci V, Wijmenga C, Troncone R, Mearin ML. Randomized feeding intervention in infants at high risk for celiac disease. N Engl J Med. 2014;371: 1304-15.

7. Andrén Aronsson C, Lee HS, Koletzko S, Uusitalo U, Yang J, Virtanen SM, Liu E, Lernmark Å, Norris JM, Agardh D, TEDDY Study Group. Effects of Gluten Intake on Risk of Celiac Disease: A Case-Control Study on a Swedish Birth Cohort. Clin Gastroenterol Hepatol. 2016;14:403-9.

8. Mårild K, Kahrs CR, Tapia G, Stene LC, Størdal K. Infections and risk of celiac disease in childhood: a prospective nationwide cohort study. Am J Gastroenterol. 2015;110:1475-84.

9. Husby S, Koletzko S, Korponay-Szabó IR, Mearin ML, Phillips A, Shamir R, Troncone R, Giersiepen K, Branski D, Catassi C, Lelgeman M, Mäki M, RibesKoninckx C, Ventura A, Zimmer KP, ESPGHAN Working Group on Coeliac Disease Diagnosis; ESPGHAN Gastroenterology Committee; European Society for Pediatric Gastroenterology, Hepatology, and Nutrition. European Society for Pediatric Gastroenterology, Hepatology, and Nutrition guidelines for the diagnosis of coeliac disease. J Pediatr Gastroenterol Nutr. 2012;54: 136-60.

10. Wolf J, Hasenclever D, Petroff D, Richter T, Uhlig HH, Laaß MW, Hauer A, Stern M, Bossuyt X, de Laffolie J, Flemming G, Villalta D, Schlumberger W, Mothes T. Antibodies in the diagnosis of coeliac disease: a biopsycontrolled, international, multicentre study of 376 children with coeliac disease and 695 controls. PLOS ONE. 2014;9:e97853.
11. Webb C, Norström F, Myléus A, Ivarsson A, Halvarsson B, Högberg L, Lagerqvist C, Rosén A, Sandström O, Stenhammar L, Carlsson A. Celiac disease can be predicted by high levels of anti-tissue transglutaminase antibodies in population-based screening. J Pediatr Gastroenterol Nutr. 2015;60:787-91.

12. Bhattacharya M, Lomash A, Sakhuja P, Dubey AP, Kapoor S. Clinical and histopathological correlation of duodenal biopsy with IgA anti-tissue transglutaminase titers in children with celiac disease. Indian J Gastroenterol. 2014;33:350-4

13. Kurppa K, Salminiemi J, Ukkola A, Saavalainen P, Löytynoja K, Laurila K, Collin $P$, Mäki M, Kaukinen $K$. Utility of the new ESPGHAN criteria for the diagnosis of celiac disease in at-risk groups. J Pediatr Gastroenterol Nutr. 2012;54:387-91.

14. Paul SP, Mazhar H, Spray CH. Applicability of the New ESPGHAN Guidelines for Diagnosing Coeliac Disease in Children from Resource Limited Countries. J Coll Physicians Surg Pak. 2015;25:455-7.

15. Oberhuber G, Granditsch G, Vogelsang H. The histopathology of coeliac disease: time for a standardized report scheme for pathologists. Eur J Gastroenterol Hepatol. 1999;11:1185-94.

16. Benelli E, Carrato V, Martelossi S, Ronfani L, Not T, Ventura A. Coeliac disease in the ERA of the new ESPGHAN and BSPGHAN guidelines: a prospective cohort study. Arch Dis Child. 2016;101:172-6.

17. Murch S, Jenkins $H$, Auth M, Bremner R, Butt A, France S, Furman M, Gillett P, Kiparissi F, Lawson M, McLain B, Morris MA, Sleet S, Thorpe M, BSPGHAN. Joint BSPGHAN and Coeliac UK guidelines for the diagnosis and management of celiac disease in children. Arch Dis Child. 2013;98:806-11.

18. Auricchio R, Tosco A, Piccolo E, Galatola M, Izzo V, Maglio M, Paparo F, Troncone R, Greco L. Potential celiac children: 9-year follow-up on a glutencontaining diet. Am J Gastroenterol. 2014;109:913-21.

\section{Submit your next manuscript to BioMed Central and we will help you at every step:}

- We accept pre-submission inquiries

- Our selector tool helps you to find the most relevant journal

- We provide round the clock customer support

- Convenient online submission

- Thorough peer review

- Inclusion in PubMed and all major indexing services

- Maximum visibility for your research

Submit your manuscript at www.biomedcentral.com/submit
) Biomed Central 